\title{
Semiclassical Structure of Chaotic Resonance Eigenfunctions
}

\author{
J. P. Keating, ${ }^{1}$ M. Novaes, ${ }^{1}$ S. D. Prado, ${ }^{2}$ and M. Sieber ${ }^{1}$ \\ ${ }^{1}$ School of Mathematics, University of Bristol, Bristol BS8 1TW, United Kingdom \\ ${ }^{2}$ Instituto de Física, Universidade Federal do Rio Grande do Sul, 91501-970 Porto Alegre, RS, Brazil
}

(Received 25 May 2006; published 13 October 2006)

\begin{abstract}
We study the resonance (or Gamow) eigenstates of open chaotic systems in the semiclassical limit, distinguishing between left and right eigenstates of the nonunitary quantum propagator and also between short-lived and long-lived states. The long-lived left (right) eigenstates are shown to concentrate as $\hbar \rightarrow 0$ on the forward (backward) trapped set of the classical dynamics. The limit of a sequence of eigenstates $\{\psi(\hbar)\}_{\hbar \rightarrow 0}$ is found to exhibit a remarkably rich structure in phase space that depends on the corresponding limiting decay rate. These results are illustrated for the open baker's map, for which the probability density in position space is observed to have self-similarity properties.
\end{abstract}

PACS numbers: 05.45.Mt, 03.65.Sq, 05.45.Df

In closed systems, the two most fundamental semiclassical properties are that the mean density of states is given by the Weyl law [1], which associates with each quantum state a Planck cell in the available region of phase space, and that in classically chaotic systems the stationary states have Wigner functions which are semiclassically uniform over the energy shell [2], in agreement with the quantum ergodicity theorem [3]. It is remarkable that it is still not known, in general, how these fundamental properties extend to open (scattering) systems.

In open systems, the lack of unitarity of the quantum propagator gives rise to nonorthogonal decaying eigenstates with complex energies (resonances), the imaginary parts of which are interpreted as decay rates. In the case of open chaotic systems, the classical mechanics is structured in phase space around fractal sets associated with trajectories that remain trapped for infinite times, either in the future (forward-trapped set $K_{+}$) or in the past (backwardtrapped set $K_{-}$). The mean density of resonances is believed (but not, in general, proved) to be determined by the fractal dimension of the invariant set $K_{0}=K_{+} \cap K_{-}$, the classical repeller. This is the fractal Weyl law [4-6]. (Note that this is different to the resonance statistics in weakly open systems, for which the size of the opening vanishes in the semiclassical limit [7].)

Much less is known about the resonance (or Gamow) eigenstates. These are important in many areas of physics [8] and chemistry [9], because they have a marked influence on observable quantities such as scattering cross sections and reaction rates (they are a component of the Siegert pseudostates basis in terms of which the scattering wave functions and $S$ matrix, for example, can conveniently be expanded [8]). Following the well established idea that in the semiclassical limit time-independent quantum properties should be related to time-independent classical sets, it is natural to expect that long-lived eigenstates of open systems should be determined by the structure of $K_{+}$and $K_{-}$. This was tested numerically for some right eigenstates of the open kicked rotator in Ref. [10], where the term "quantum fractal eigenstates" was coined.
We here significantly extend the notion of quantum fractal eigenstates in several new directions. First, we draw the important distinctions between left and right eigenstates of the nonunitary propagator and between states that are "short-lived" and "long-lived" with respect to the Ehrenfest time. Second, we show that in the semiclassical limit the long-lived left eigenstates concentrate on $K_{+}$, while the long-lived right eigenstates concentrate on $K_{-}$. In chaotic systems, the eigenstates thus inherit the intricate fractal structure of the underlying classical trapped sets (this property has also been observed by Nonnenmacher and Rubin [11]). Third, we find that in the semiclassical limit the eigenstates have a rich structure that reflects the self-similarity of the sets $K_{ \pm}$and depends explicitly on the limiting decay rate. A semiclassical formula is derived for the weights of the quantum eigenstates in different regions of phase space. This formula is consistent with the numerical results of Ref. [10] in the special case when the decay rate is equal to the classical escape rate.

We illustrate our results for an open quantum baker's map. In this system, we have, in addition, that in the semiclassical limit long-lived right eigenstates have fractal support in momentum space and are self-similar in position space (and vice versa for left eigenstates). Finally, we discuss a system, the Walsh-quantized baker's map, for which exact results corroborate our arguments.

Many recent studies of open quantum systems have focused on maps of the torus $T$, viewed as a phase space, which is opened by removing a strip parallel to the momentum direction [5,12-15]. A physical motivation is the "bounce map" defined for billiards, in which case the opening corresponds to attaching a perfect lead to the billiard. The quantization of the classical map $\mathcal{U}$ is a unitary matrix $U$, acting on a Hilbert space of dimension $N$. For torus maps, this dimension plays the role of an effective Planck's constant $\hbar=(2 \pi N)^{-1}$, and the semiclassical limit thus corresponds to $N \rightarrow \infty$. If the opening $\mathcal{O}$ occupies a fraction $M / N$ of the total area in phase space, then quantum mechanically the open map corresponds to a nonunitary matrix $\tilde{U}=U \Pi$, where $\Pi$ is a projector onto 
the complement of the opening. The result of multiplying by $\Pi$ is to set $M$ columns of $U$ equal to zero. Since the matrix $\tilde{U}$ is not unitary, we must distinguish between its left and right eigenstates:

$$
\tilde{U}\left|\Psi_{n}^{R}\right\rangle=z_{n}\left|\Psi_{n}^{R}\right\rangle, \quad\left\langle\Psi_{n}^{L}\right| \tilde{U}=z_{n}\left\langle\Psi_{n}^{L}\right| .
$$

We will assume $\left\langle\Psi_{n}^{L} \mid \Psi_{n}^{L}\right\rangle=\left\langle\Psi_{n}^{R} \mid \Psi_{n}^{R}\right\rangle=1$. The eigenvalues $z_{n}$ lie inside the unit circle in the complex plane $\left|z_{n}\right|^{2}=$ $e^{-\Gamma_{n}} \leq 1$, where $\Gamma_{n} \geq 0$ is the decay rate. (Note that these are not scattering states, for which fractality has also been observed [16].)

If $\mathcal{O}_{m}=\mathcal{U}^{m}(\mathcal{O})$ denotes the $m$ th image of the opening under the classical map, the forward-trapped and backward-trapped sets are defined, respectively, as

$$
K_{+}=T \backslash \bigcup_{m=0}^{\infty} \mathcal{O}_{-m}, \quad K_{-}=T \backslash \bigcup_{m=1}^{\infty} \mathcal{O}_{m} .
$$

Let us also define the set of points which fall into the opening after $m$ steps, but not earlier:

$$
\mathcal{R}_{+}^{m}=\left\{x \in \mathcal{O}_{-m}, x \notin \mathcal{O}_{-n}, 0 \leq n<m\right\} .
$$

We also define $\mathcal{R}_{-}^{m}=\left\{x \in \mathcal{O}_{m}, x \notin \mathcal{O}_{n}, 1 \leq n<m\right\}$ for $m>1$ and $\mathcal{R}_{-}^{1}=\mathcal{O}_{1}$. These sets are related by

$$
\mathcal{U}^{-1}\left(\mathcal{R}_{+}^{m}\right) \backslash \mathcal{O}=\mathcal{R}_{+}^{m+1}, \quad \mathcal{U}\left(\mathcal{R}_{-}^{m} \backslash \mathcal{O}\right)=\mathcal{R}_{-}^{m+1},
$$

with the convention that $\mathcal{R}_{+}^{0}=\mathcal{O}$.

The Ehrenfest time $\tau_{E}$ plays an important role in quantum chaotic transport [13-15,17], essentially determining the quantum-to-classical crossover. This is the time it takes for a minimal wave packet to become larger than the opening, being therefore partially reflected when leaving the system. For times shorter than $\tau_{E}$, the quantum evolution of localized states can be approximated by the classical evolution. For open chaotic maps, $\tau_{E}=\lambda^{-1} \ln M$, where $M$ is the number of "open channels" and $\lambda$ is the Lyapunov exponent $[14,15]$. In the semiclassical limit, quantum states that are essentially supported on some region $\mathcal{A} \subset \mathcal{R}_{+}^{n}$ of phase space will have a deterministic escape and will thus become eigenstates of $\tilde{U}^{n+1}$ corresponding to eigenvalues that vanish as $\hbar \rightarrow 0$. These are called short-lived states $[14,15]$. The estimate that the fraction of such states is $1-e^{-\tau_{E} / \tau_{D}}$, which holds when the mean dwell time $\tau_{D}=N / M$ is large, results in the fractal Weyl law [15]. Short-lived states are intimately related to the short-time dynamics and are relatively insensitive to the classical trapped sets. In what follows, we obtain several results for the semiclassical limit of longlived eigenstates.

One way to look at the eigenstates is to depict them in phase space using the Husimi representation, in which one associates with any eigenstate $\left|\Psi_{n}^{\xi}\right\rangle(\xi=L, R)$ the function $H_{n}^{\xi}(x)=\left|\left\langle x \mid \Psi_{n}^{\xi}\right\rangle\right|^{2}$, where $x=(q, p)$ is a point of phase space and $|x\rangle$ is a coherent state. From (1), we have for the Husimi function of a right eigenstate

$$
\left|z_{n}\right|^{2 m}\left|\left\langle x \mid \Psi_{n}^{R}\right\rangle\right|^{2}=\left|\left\langle x\left|\tilde{U}^{m}\right| \Psi_{n}^{R}\right\rangle\right|^{2} .
$$

For times much shorter than the Ehrenfest time, the action of the quantum propagator on coherent states can be approximated by the classical evolution; in particular, we have that as $\hbar \rightarrow 0$

$$
\left(\tilde{U}^{\dagger}\right)^{m}|x\rangle \approx 0 \quad \text { if } x \in \mathcal{R}_{-}^{m} .
$$

Therefore, in the semiclassical limit $H_{n}^{R}$ becomes negligible on regions $\mathcal{R}_{-}^{m}$ if $\left|z_{n}\right|>0$ is fixed and $m \ll \tau_{E}$. Since the Ehrenfest time grows as $\hbar \rightarrow 0$, the function must concentrate on the backward-trapped set $K_{-}$in this limit,

$$
H_{n}^{R}(x) \approx 0 \quad \text { if } x \notin K_{-} \quad\left(\left|z_{n}\right|>0, \quad \hbar \rightarrow 0\right) .
$$

For a finite $\hbar$, there will be some leakage of $\left|\left\langle x \mid \Psi_{n}^{R}\right\rangle\right|^{2}$ into the regions $\mathcal{O}_{m}$, mostly for large $m$. This deviation should be more pronounced for states with a small lifetime. The reasoning for long-lived left eigenstates is completely analogous. In the semiclassical limit, they concentrate on the forward-trapped set $K_{+}$.

For finite $\hbar$, the Husimi functions are not supported on truly fractal sets, but as $\hbar \rightarrow 0$ finer classical structures are revealed. Continuous time Hamiltonian systems can be treated in much the same way. One just replaces $\left|z_{n}\right|^{2 m}$ by $e^{-\Gamma_{n} T}$, and the essence of the argument remains. We thus expect Husimi functions that live on fractal sets in generic open chaotic systems in the semiclassical limit.

Let us investigate the weight of the eigenstates on different regions of phase space. Let $\pi_{0}$ be the projector onto the opening. Our nonunitary propagator then satisfies $\tilde{U}^{\dagger} \tilde{U}=$ $1-\pi_{0}$. Hence, we find that the weight of the right eigenstates in the opening

$$
\left\langle\Psi_{n}^{R}\left|\pi_{0}\right| \Psi_{n}^{R}\right\rangle=1-\left|z_{n}\right|^{2}
$$

increases with increasing decay rate. Let $\pi_{m}, m \geq 1$, be the projector onto $\mathcal{R}_{+}^{m}$ (this is an example of the general class of projectors introduced in Ref. [18]). For short times, the semiclassical approximation gives $\tilde{U}^{\dagger} \pi_{m} \tilde{U} \approx \pi_{m+1}$, and thus we obtain the remarkably simple relation

$$
\left\langle\Psi_{n}^{R}\left|\pi_{m}\right| \Psi_{n}^{R}\right\rangle \approx\left|z_{n}\right|^{2 m}\left(1-\left|z_{n}\right|^{2}\right) .
$$

Notice that this vanishes for any fixed $m$ as $\left|z_{n}\right| \rightarrow 1$; thus, if the quantum decay rate approaches zero as $\hbar \rightarrow 0$, the corresponding eigenstate becomes localized on the invariant set $K_{0}$.

It is interesting to note that the distribution of the eigenstates on phase space depends explicitly on the corresponding decay rate, which we hold fixed as $\hbar \rightarrow 0$. This is an important difference with respect to the case of unitary evolution, when the semiclassical limit is the same for almost all sequences of eigenstates $\{\psi(\hbar)\}_{\hbar \rightarrow 0}$. We also note that the areas of the regions $\mathcal{R}_{+}^{m}$ are proportional to $e^{-m \gamma_{c}}$, where $\gamma_{c}$ is the classical escape rate. Therefore, the prediction in Ref. [10] that a right eigenstate of an open 
quantum map with $\Gamma_{n} \approx \gamma_{c}$ should be constant (up to quantum fluctuations) over the classical set $K_{-}$is consistent with our expression.

We now illustrate our results in a paradigmatic model of quantum chaos, the baker's map, which is particularly convenient because of the relatively simple structure of its trapped sets. Its triadic form is defined as

$$
\mathcal{U}(q, p)= \begin{cases}\left(3 q, \frac{p}{3}\right) & \text { if } 0 \leq q<\frac{1}{3}, \\ \left(3 q-1, \frac{p+1}{3}\right) & \text { if } \frac{1}{3} \leq q<\frac{2}{3}, \\ \left(3 q-2, \frac{p+2}{3}\right) & \text { if } \frac{2}{3} \leq q<1\end{cases}
$$

This system is uniformly hyperbolic, with Lyapunov exponent $\lambda=\ln 3$. The stable and unstable manifolds are parallel to the momentum and position axes, respectively. Its quantization is given by [19]

$$
U_{N}=F_{N}^{-1} \operatorname{diag}\left(F_{N / 3}, F_{N / 3}, F_{N / 3}\right),
$$

where the subscript denotes the dimension of the matrix. $F_{N}$ is a modified Fourier transform $\left(F_{N}\right)_{n m}=$ $(1 / \sqrt{N}) e^{-(2 \pi i / N)(n+1 / 2)(m+1 / 2)}$, with the integers $n$ and $m$ running from 0 to $N-1$. The " $1 / 2$ " factors are necessary in order to impose parity on the eigenstates. This form of the matrix $F_{N}$ leads to antiperiodic boundary conditions.

An open version of this system was introduced in Refs. [5,12], in the context of the fractal Weyl law, where the opening was taken as the middle vertical strip (see also Ref. [20], where a closely related map was studied). To find the forward-trapped set $K_{+}$, one may consider the backward propagation of this strip, and it is not difficult to see that the only points that remain are those belonging to Can $\times[0,1)$, where "Can" denotes the usual middle-third fractal Cantor set. The backward-trapped set $K_{-}$is $[0,1) \times$ Can. Details of this construction can be found in Refs. [5,12]. The open quantum system is obtained by setting the middle third of the columns of $U_{N}$, corresponding to the strip, equal to zero. The kinematics is such that the position representation of right eigenstates is equal to the momentum representation of left eigenstates.

In Fig. 1, we plot the average of $H_{n}^{R}(q, p)$ over the 100 longest-lived states, for the case $N=3^{7}$. We see that it is reasonably concentrated on the backward-trapped set, although it is not able to resolve this set on the finest scales. More details of $K_{-}$should be revealed for larger values of $N$. The right panel in Fig. 1 shows the averaged Wigner function, and we see that it resolves $K_{-}$with considerably greater accuracy.

In Fig. 2, we plot the weight of right eigenstates on the regions $\mathcal{R}_{+}^{m}$, for $N=3^{6}$. The solid lines represent the semiclassical approximation (9). We see that the agreement is rather good, particularly for small eigenvalue modulus and small values of $m$.

Let us now turn our attention to wave functions in position or momentum space. For the baker's map, the projection of $K_{-}$onto momentum space is just the Cantor set, and the momentum representation of any
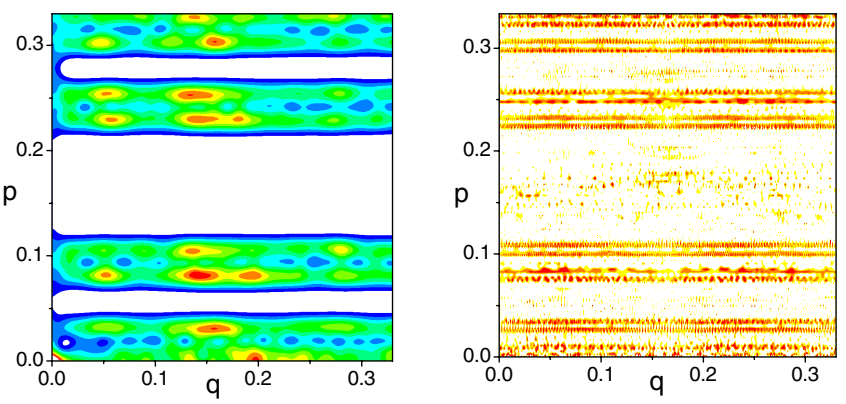

FIG. 1 (color online). Left panel: The average of the Husimi functions of the 100 longest-lived right eigenstates of the baker's map, for $N=3^{7}$ (intensity increases from blue to red). Right panel: The corresponding Wigner function average (in the white regions, the function is nonpositive). Note that the $\hbar$ scale is $1 / N \approx 0.005$.

long-lived right eigenstate must be supported on this fractal dust. In Fig. 3, we plot $\left|\Psi^{R}(p)\right|^{2}$, averaged over the 25 longest-lived states, for $N=3^{7}$. The fractal nature of the support, within the $\frac{1}{N}$ resolution set by the uncertainty principle, is evident from the magnification by a factor of 3. In Fig. 4, we plot $\left|\left\langle q \mid \Psi^{R}\right\rangle\right|^{2}$ averaged over a few right eigenstates, again for $N=3^{7}$. Figures 4 (a) and 4(b) correspond to a relatively large decay rate, while Figs. 4(c) and 4 (d) correspond to a smaller one. Within the available resolution, both functions are remarkably self-similar, as can be appreciated from the magnifications. This observation is consistent with our theory [cf. (9)].

Nonnenmacher and Zworski have recently introduced a simplified version of the open quantum baker's map, based on the "Walsh-Fourier" transform, that can be solved explicitly [12]. For this system, several of the results presented here are exact and can be rigorously proven. One particular feature is that the difference between short-lived and long-lived states is well defined. The former all have exactly null eigenvalue, and the phase space representation of the latter vanishes outside the finitely resolved relevant trapped set. Equations (6) and (9) are exact for this system. We have also shown that self-similar properties analogous to those illustrated by Fig. 4 can be
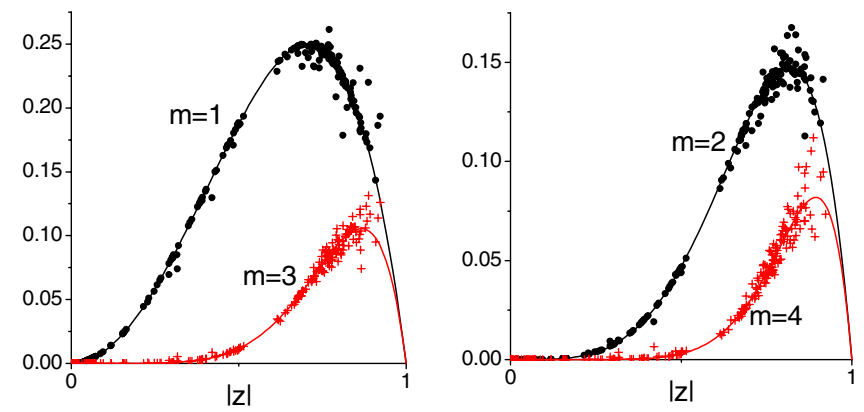

FIG. 2 (color online). The weight $\left\langle\Psi_{n}^{R}\left|\pi_{m}\right| \Psi_{n}^{R}\right\rangle$ of right eigenstates of the baker's map on the regions $\mathcal{R}_{+}^{m}$, for $N=3^{6}$. The solid line is the semiclassical approximation (9). 

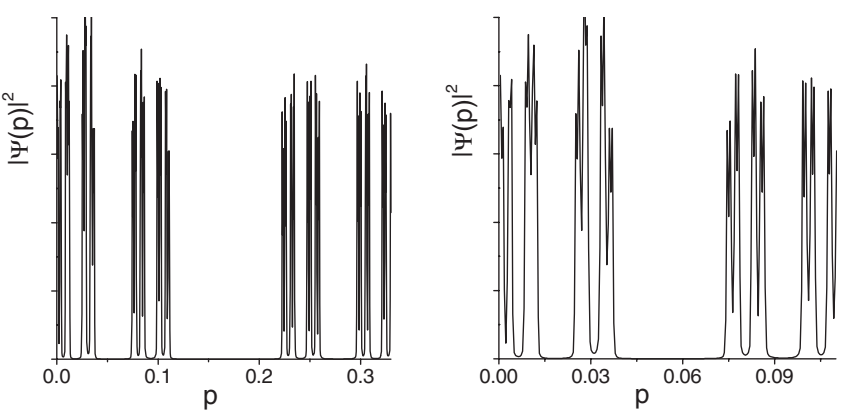

FIG. 3. Probability density in momentum space for right eigenstates $\left|\left\langle p \mid \Psi^{R}\right\rangle\right|^{2}$, averaged over the 20 longest-lived states (eigenvalue modulus ranging from 0.90 to 0.83 ). This function is approximately supported on the Cantor set, as can be seen from a magnification by a factor of 3 (right panel). $N=3^{7}$ and so the $\hbar$ scale is $1 / N \approx 0.005$.

established rigorously and that - after either a spatial average or a spectral average - the probability density of long-lived right eigenstates in the momentum representation becomes constant on the Cantor set as $N \rightarrow \infty$ (for the standard quantization, this is suggested by Fig. 3). A detailed account will be presented elsewhere [21].

In summary, we have shown that in the semiclassical limit resonance eigenstates of open chaotic systems concentrate on classical fractal trapped sets. We have derived a formula for their distribution on different areas of phase space that depends explicitly on the quantum decay rate.
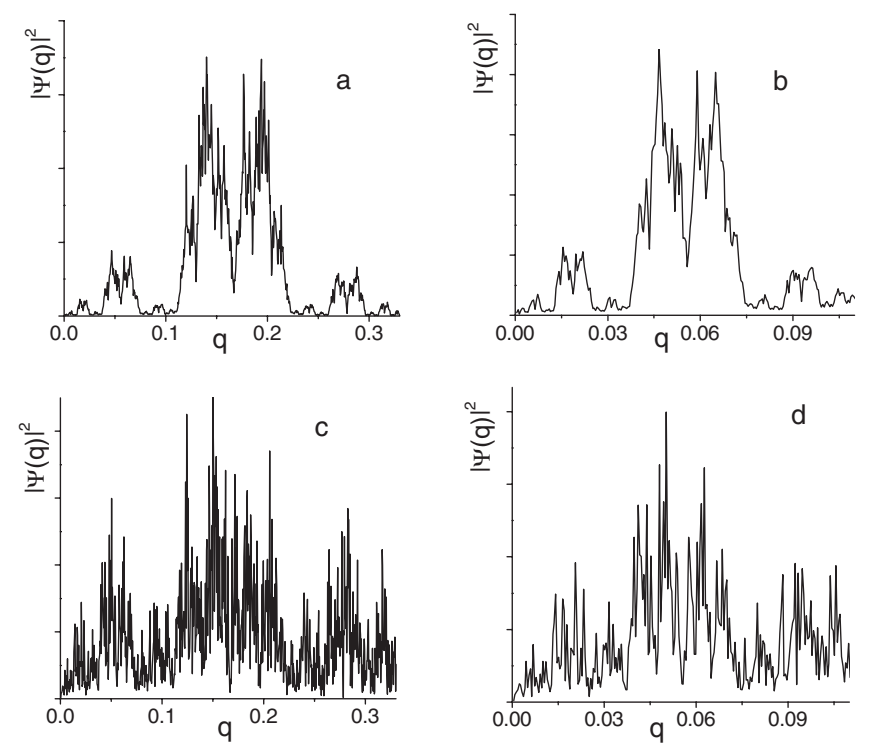

FIG. 4. Probability density in position space for right eigenstates $\left|\left\langle q \mid \Psi^{R}\right\rangle\right|^{2}$, averaged over states with similar decay rates. (b) and (d) are magnifications of (a) and (c). The average eigenvalue modulus is approximately 0.4 for (a),(b) and 0.7 for (c),(d). In both cases, the self-similarity is striking. $N=3^{7}$.
For the baker's map, we have also found that the position and momentum representations of wave functions exhibit self-similarity.

This research was supported by EPSRC and the Royal Society. One of the authors (M.N.) also gratefully acknowledges financial support from CAPES.

[1] M. Dimassi and J. Sjöstrand, Spectral Asymptotics in the Semiclassical Limit (Cambridge University Press, Cambridge, England, 1999).

[2] M. V. Berry, J. Phys. A 10, 2083 (1977).

[3] A. I. Shnirelman, Usp. Mat. Nauk 29, 181 (1974).

[4] J. Sjöstrand, Duke Math. J. 60, 1 (1990); M. Zworski, Not. Am. Math. Soc. 46, 319 (1999).

[5] J. Sjöstrand and M. Zworski, math.SP/0506307.

[6] K. K. Lin, J. Comput. Phys. 176, 295 (2002); K. K. Lin and M. Zworski, Chem. Phys. Lett. 355, 201 (2002); W. T. Lu, S. Sridhar, and M. Zworski, Phys. Rev. Lett. 91, 154101 (2003).

[7] Y. V. Fyodorov and H.-J. Sommers, J. Math. Phys. (N.Y.) 38, 1918 (1997); J. Phys. A 36, 3303 (2003).

[8] O. I. Tolstikhin, V. N. Ostrovsky, and H. Nakamura, Phys. Rev. Lett. 79, 2026 (1997); Phys. Rev. A 58, 2077 (1998); G. V. Sitnikov and O. I. Tolstikhin, ibid. 67, 032714 (2003).

[9] B. K. Kendrick et al., Phys. Rev. Lett. 84, 4325 (2000); R. T. Skodje et al., J. Chem. Phys. 112, 4536 (2000); G. C. Schatz, Science 288, 1599 (2000);

[10] G. Casati, G. Maspero, and D. L. Shepelyansky, Physica (Amsterdam) 131D, 311 (1999).

[11] S. Nonnenmacher and M. Rubin (to be published).

[12] S. Nonnenmacher and M. Zworski, J. Phys. A 38, 10683 (2005); see also math-ph/0505034.

[13] P. G. Silvestrov, M. C. Goorden, and C. W. J. Beenakker, Phys. Rev. B 67, 241301 (2003); J. Tworzydlo et al., ibid. 68, 115313 (2003); Ph. Jacquod and E. V. Sukhorukov, Phys. Rev. Lett. 92, 116801 (2004); S. Rahav and P. W. Brouwer, ibid. 95, 056806 (2005); 96, 196804 (2006); Ph. Jacquod and R.S. Whitney, Phys. Rev. B 73, 195115 (2006).

[14] H. Schomerus and Ph. Jacquod, J. Phys. A 38, 10663 (2005).

[15] H. Schomerus and J. Tworzydlo, Phys. Rev. Lett. 93, 154102 (2004).

[16] H. Ishio and J. P. Keating, J. Phys. A 37, L217 (2004).

[17] I. Aleiner and A. Larkin, Phys. Rev. B 54, 14423 (1996); S. Oberholzer, E. V. Sukhorukov, and C. Schonenberger, Nature (London) 415, 765 (2002).

[18] M. Saraceno and A. Voros, Physica (Amsterdam) 79D, 206 (1994); R. O. Vallejos and M. Saraceno, J. Phys. A 32, 7273 (1999).

[19] N.L. Balazs and A. Voros, Ann. Phys. (N.Y.) 190, 1 (1989); M. Saraceno, ibid. 199, 37 (1990).

[20] M. Saraceno and R. O. Vallejos, Chaos 6, 193 (1996).

[21] J.P. Keating et al. (to be published). 